\title{
Screw Intramedullary Nailing for Fractures of the Humeral Shaft
}

\author{
Y. S. Salphale*, W. M. Gadegone, R. M. Chandak, Jayeshkumar Dave \\ Department of Orthopaedics \& Traumatology, Chandrapur Multispeciality Hospital, Chandrapur, India \\ Email: *yogeshsalphale@gmail.com
}

Received 27 June 2015; accepted 16 August 2015; published 19 August 2015

Copyright (C) 2015 by authors and Scientific Research Publishing Inc.

This work is licensed under the Creative Commons Attribution International License (CC BY). http://creativecommons.org/licenses/by/4.0/

(c) (i) Open Access

\begin{abstract}
The debate continues over the management of diaphyseal fractures of the humerus. There are a variety of extramedullary as well as intramedullary implants. We aim to propose a technique of passing the screw intramedullary nails and achieve union with least trauma to the shoulder and the rotator cuff. The multiple elastic screw nails achieve the inherent stability based on the principle of "three point fixation". We aim to propose that the screw intramedullary nail is an effective implant to facilitate uneventful fracture union, with rapid recovery, low morbidity and low learning curve capable of being replicated in any smaller operative set up.
\end{abstract}

\section{Keywords}

Fracture Humerus, Diaphyseal Humeral Fracture, Screw Intramedullary Nail, Humerus Nailing

\section{Introduction}

The humeral shaft fractures can be managed by closed reduction and casting as well as by a variety of surgical means. The results are equally encouraging.

It is paradox why a surgical technique, so successful in the treatment of diaphyseal femoral and tibial fractures cannot produce similar results when applied in the humerus. A possible explanation is that the complex anatomy and the unique biomechanical characteristics of the humerus are often overlooked.

However with the advent of new tools and techniques the armamentarium of a trauma surgeon is enriched. Open reduction and internal fixation with plates give good radiological reduction but are fraught with complications like infection and radial nerve palsy [1] [2]. We aim to propose a technique, never used for the fractures of the humeral shaft, after seeking approval from the hospital ethics committee which could be undertaken at any district level hospital, with minimum instrumentation with a relatively low learning curve.

${ }^{*}$ Corresponding author. 
The screw intramedullary nail (manf. K-AIMS ORTHO Implants, Mumbai, India) is a titanium nail available in diameter of 2, 3, and $4 \mathrm{~mm}$ with bevelled tip. A threaded head is blended with the nail, which is positioned at the end of nail held in place by circular running notch located on the end of nail shaft. This design allows the self-cutting thread approximately $20 \mathrm{~mm}$ to be advanced and screwed in with a $3.5 \mathrm{~mm}$ screw driver. The distal bevelled end of the nail aids in fracture reduction and helps in engaging in the subchondral area of the bone, thereby imparting stability (Figure 1 and Figure 2). By gently screwing with a $3.5 \mathrm{~mm}$ screw driver the nail is adequately buried into the proximal metaphyseal region.

\section{Case Report}

A thirty-seven years old male sustained a fracture of the middiaphysis of the right humeral shaft following a road accident Figure 3. It was a closed injury and there was no neurovascular deficit.

Surgery was performed within seventy two hours, on a standard operation table under the guidance of image intensifier. Inj. Cefuroxime axetil 1.5 gms was given before the induction of anaesthesia and two doses later on in the interval of 12 hours. The patient was placed in a supine position. A sandbag draped in sterile cloth was placed under the affected scapula. The neck was turned to the opposite side and the arm was abducted to approximately 20 degrees. Closed reduction was carried out and ascertained by image intensifier on anteroposterior and lateral views.

Reduction of the fracture is usually achieved by a combination of adduction (arm against chest), neutral forearm rotation (forearm straight up toward ceiling), and traction. An assistant is necessary to maintain this reduction during nail insertion.

The greater tuberosity was located and a $1 \mathrm{cms}$ incision distal to it was taken. With a sharp pointed awl an

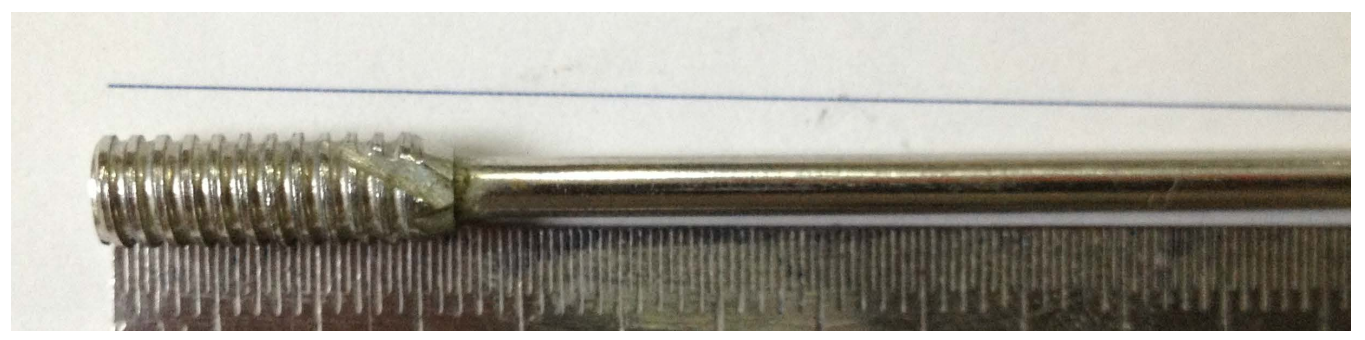

Figure 1. Showing the profile of the implant which engages the proximal humerus.

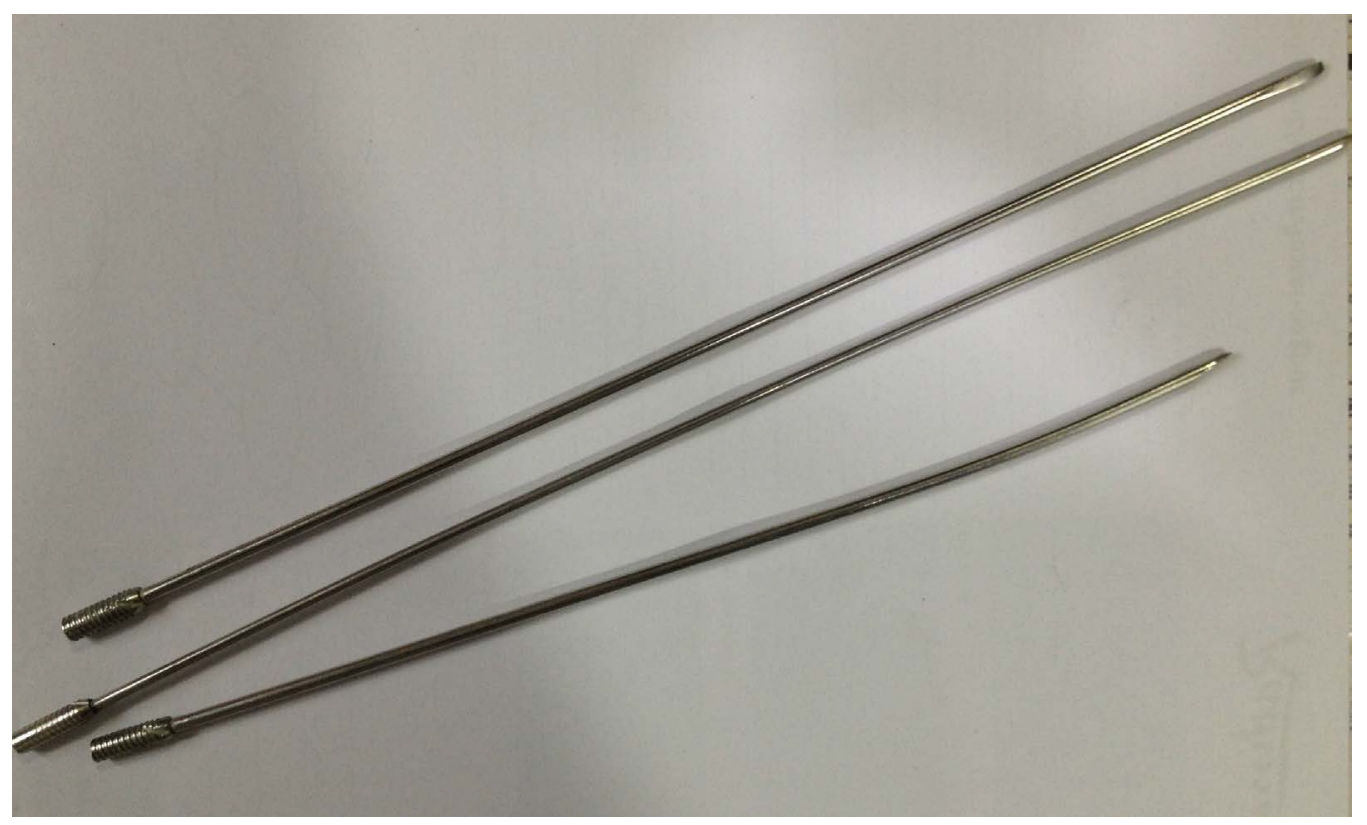

Figure 2. Showing the screw intramedullary nail with a beveled tip. 


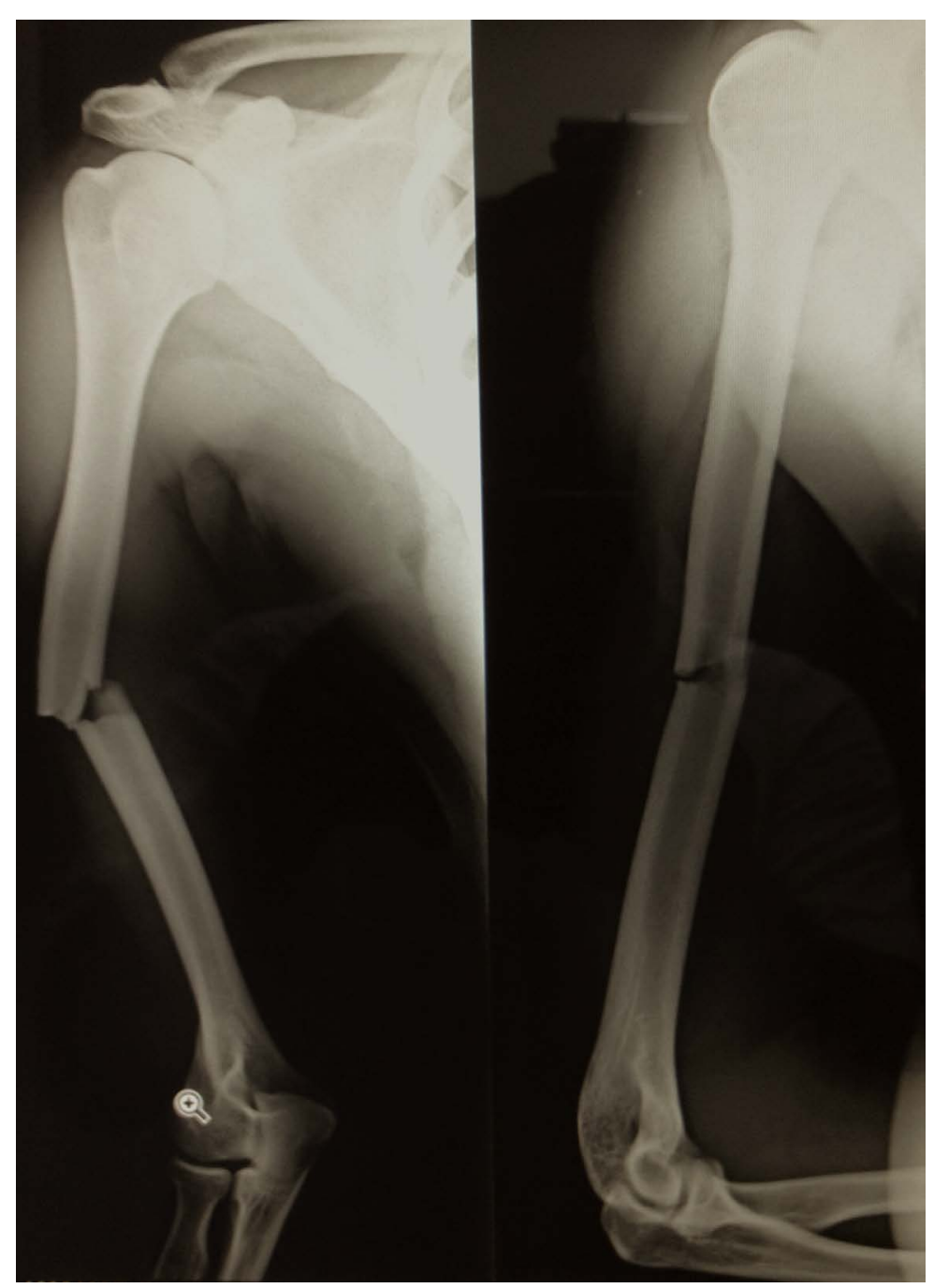

Figure 3. Showing fracture of the humeral shaft.

entry point was made. The screw intramedullary nail of adequate diameter and predetermined length is advanced gradually till it reached the distal metaphyseal region of the humerus. Two $1 \mathrm{~cm}$ incisions were taken anteriorly and posteriorly from the primary incision and nails were inserted (Figure 4).

The nail is driven below the cortical surface of the humeral head to prevent subacromial impingement and rotator cuff irritation (Figure 5). Multiple fluoroscopic images of the arm in various positions must be obtained to confirm that the nail is below the horizon of the humeral head and adequately countersunk by a $3.5 \mathrm{~mm}$ screw driver.

The medullary cavity was filled with screw intramedullary nails of unequal lengths, in the sizes ranging from $2 \mathrm{~mm}$ to $4 \mathrm{~mm}$. A minimum of two and maximum of three screw intramedullary nails could be negotiated within the medullary Canal. The shoulder was moved in all the direction to check the possible obstruction by the nails. Care was taken to avoid distraction at the fracture site. The mean duration for the surgery was 35 minutes. Postoperatively a strapping was given for a period of three weeks. Active and passive exercises were initiated as soon as the pain tolerance of the patient improved (Figure 6).

The minimal incidence of the shoulder pain in our series could be attributed to the small incision and the atraumatic technique sparing the rotator cuff.

\section{Discussion}

The successful treatment of humeral fractures depends on many factors; the age of the patient, the patients 


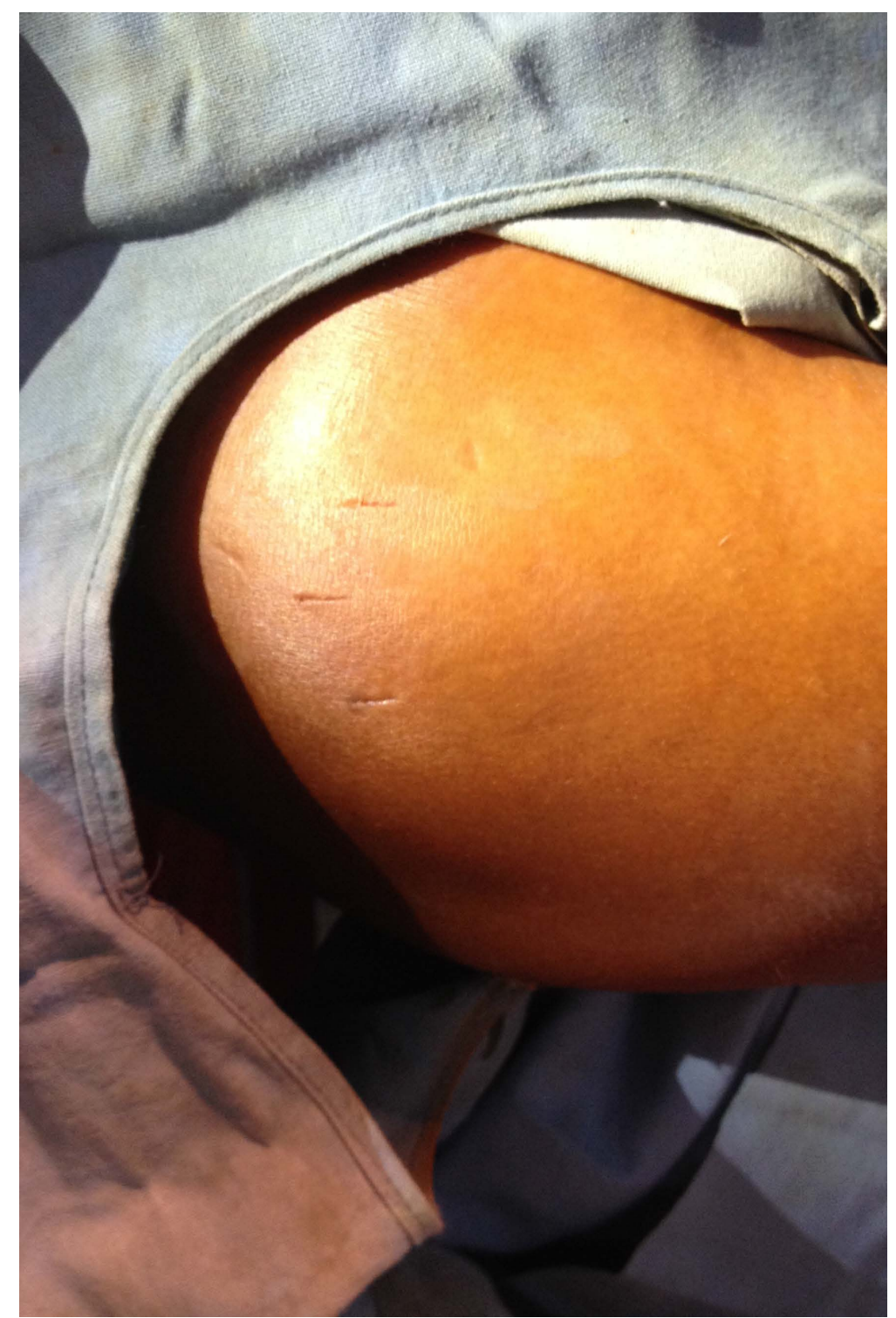

Figure 4. Showing the patient on the operating table with the incisions marked showing fracture shaft humerus.

general health, the time from fractures to treatment, the adequacy of treatment, concurrent medical history, the adequacy of treatment and stability of fixation.

IM nails can be placed without direct fracture exposure and with much less soft tissue dissection. Additionally, cortical osteopenia caused by stress shielding as seen with plates and screws is less likely noted [3].

Violation of the rotator cuff during antegrade humeral nailing has been considered to be responsible for suboptimal clinical outcomes and discomfort in the shoulder joint [4]-[7]. Retrograde nailing was mainly introduced as an alternative technique to bypass this problem [8]-[10].

Proximal and distal locking are integral elements for rotational stability of an IMN. There are unique issues with the distal humerus especially when attempting to get a good lateral view and localising the narrow distal locking holes. The lateral surface of the humerus is "slippery" and the danger of injury to the radial nerve and other vulnerable structures exists if adequate care is not exercised [11]-[13]. The success of commercial targeting devices is still inconsistent.

The location for entry hole is subject to debate. The linear acces to humeral IM canal is possible only through a portal made in the sulcus between the greater tuberosity and the articular surface. 


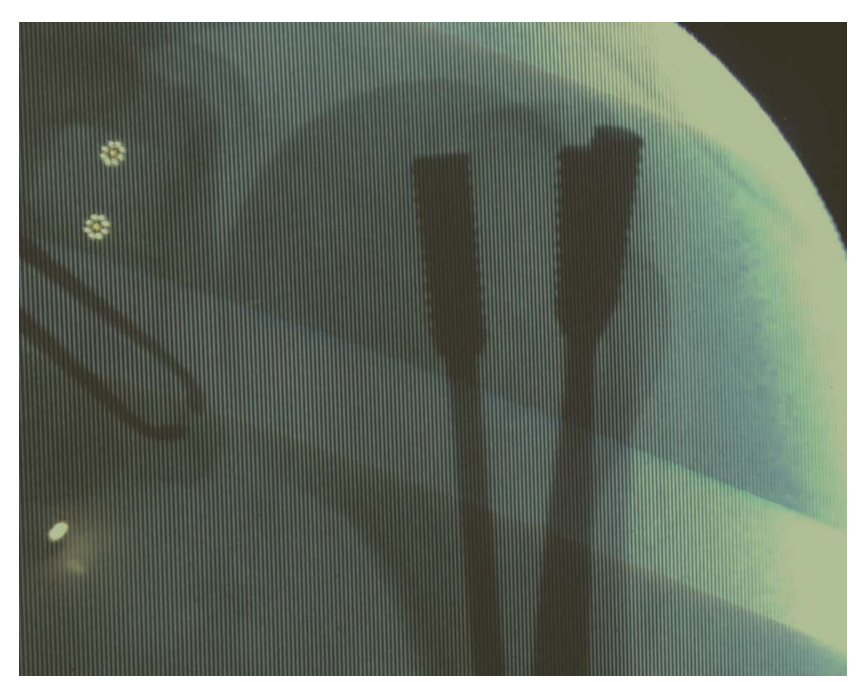

Figure 5. C arm confirmation of the buried nails.

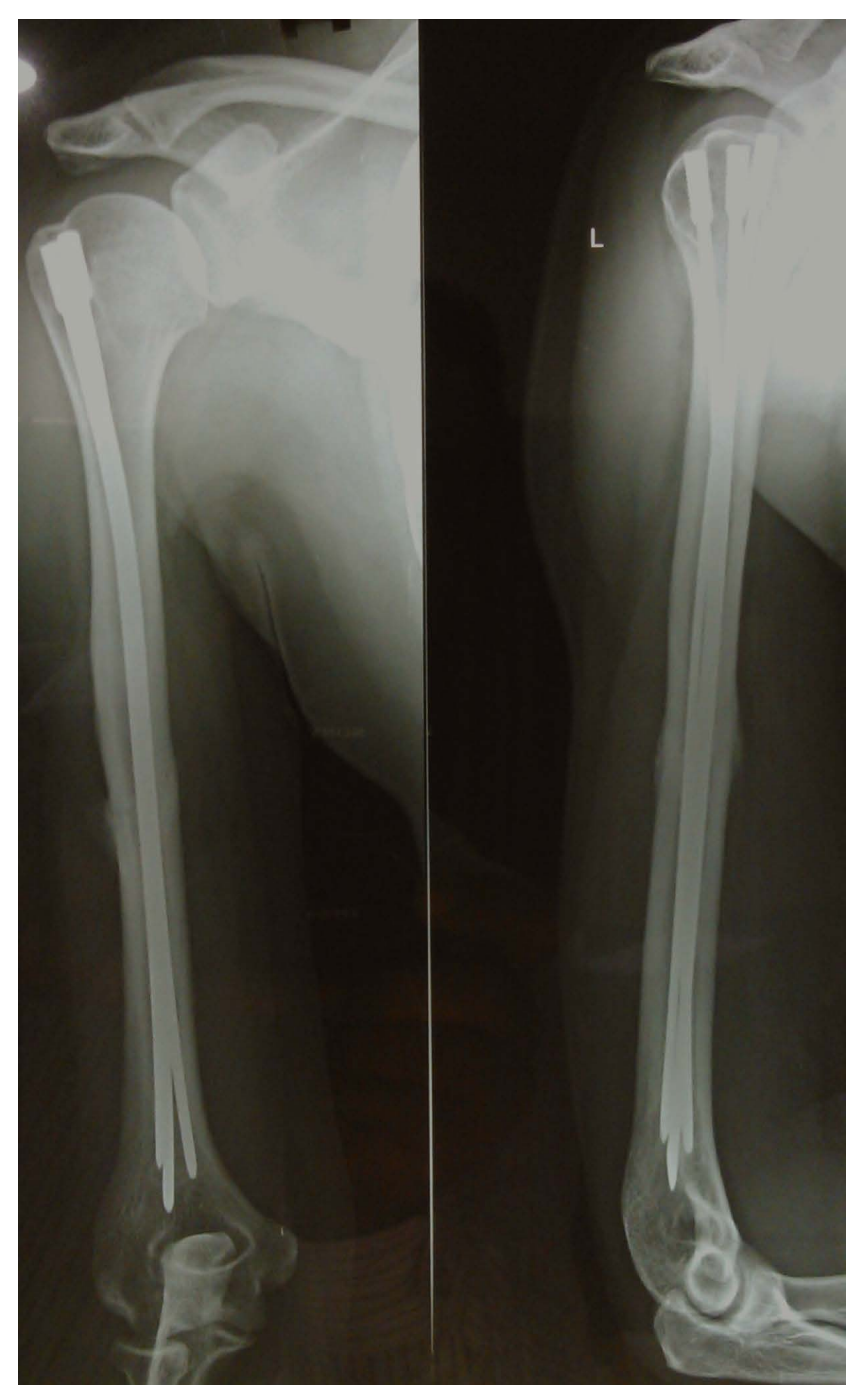

Figure 6. Showing well seated screw intramedullary nail and a healed fracture. 
Since the surgical procedure in antegrade humeral nailing requires a split of the supraspinatus tendon, the question arises whether the surgical approach causes microcirculatory dysfunction of the tendon [14]. Antegrade nailing of proximal humeral fractures using a straight nail can damage the bony insertion of the supraspinatus tendon leading to varus failure of the construct [15].

Open reduction and internal fixation (ORIF) with a plate and screws provides direct fracture exposure and near anatomic alignment. The rates of nonunion and hardware failure requiring revision range from $0 \%-7 \%$ [16].

The inherent stability achieved by the screw intramedullary nailing, is based on the principle of "three point fixation". The screw intramedullary nailing rests on the principle of stacking the nails in the waist of the medullary cavity spreading the bunch of nails in the metaphysis thereby providing better axial, angulatory and rotational stability. At the proximal end the screw end of the nails are flush with the articular surface. This helps in removal of the implant, should the need arises. Care should be exercised to prevent aggressive advancement of the implant as the screw threads can get buried in the proximal cancellous portion of the humeral head causing difficulty in extracting the implants.

The ante grade insertion is known to have a fewer problems than the retrograde technique if the rotator cuff is not violated [17].

By resorting to the small incisons and getting access into the medullary canal of the proximal humerus we feel that the traumatic insult to the cuff is minimised. Moreover the fanning and converging of the nails provides a good anchorage and stability.

The attractive theoretical advantages of locking humeral nails have not been borne out in clinical studies. Complications such as shoulder pain, delayed union or nonunion, fracture about the implant, iatrogenic fracture communition, and the difficulty in the reconstruction of failures have diminished their usefulness [18].

The screw intramedullary nail obviates the difficulties of proximal and distal locking and the operative radiation.

We placed our incision $1 \mathrm{~cm}$ below the crest of the greater tuberosity, in a region outside the articular surface and rotator cuff area thereby avoiding a significant trauma [19].

Unlike the other long bones, distraction is poorly tolerated by the Humerus. Care should be taken to avoid it and a splintage (strapping) helps in preventing the possible distraction during the period of healing.

Other weak points include the potential failure of fixation stability, and the restriction in postoperative mobilization.

\section{Summary}

The screw intramedullary nail seems to be a good technique of fixation with low risk of infection, minimal cost, promoting faster healing because the blood supply is only altered insignificantly. No preparation of the medullary cavity is necessary for implantation. Moreover the implant placed flush with bone causes less damage to soft tissue at nail entry point.

We conclude that the closed medullary fixation by multiple screw intramedullary nails has an edge over the other fixation methods in terms of mechanical and biological advantages.

\section{References}

[1] Foster, R.J., Dixon Jr., G.L., Bach, A.W., et al. (1985) Internal Fixation of Fractures and Non-Unions of the Humeral Shaft. Indications and Results in a Multi-Center Study. Journal of Bone \& Joint Surgery, American Volume, 67, 857864.

[2] Rodriguez-Merchan, E.C. (1995) Compression Plating versus Hackethal Nailing in Closed Humeral Shaft Fractures Failing Non Operative Reduction. Journal of Orthopaedic Trauma, 9, 194-197. http://dx.doi.org/10.1097/00005131-199506000-00003

[3] Beaty, J.H. (1999) Humeral Shaft Fractures. In: Orthopaedic Knowledge Update, American Academy of Orthopaedic Surgeons, Rosemont, 278-286.

[4] Wallny, T., Sagebiel, C., Westerman, K., Wagner, U.A. and Reimer, M. (1997) Comparative Results of Bracing and Interlocking Nailing in the Treatment of Humeral Shaft Fractures. International Orthopaedics, 21, 374-379. http://dx.doi.org/10.1007/s002640050189

[5] Stern, P.J., Mattingly, D.A., Pomeroy, D.L., Zenni Jr., E.J. and Kreig, J.K. (1984) Intramedullary Fixation of Humeral 
Shaft Fractures. Journal of Bone \& Joint Surgery, American Volume, 66, 639-646.

[6] Brumback, R.J., Bosse, M.J., Poka, A. and Burgess, A.R. (1986) Intramedullary Stabilization of Humeral Shaft Fractures in Patients with Multiple Trauma. Journal of Bone \& Joint Surgery, American Volume, 68, 960-969.

[7] Riemer, B.L., D’Ambrosia, R., Kellam, J.F., Butterfield, S.L. and Burke 3rd, C.J. (1993) The Anterior Acromial Approach for Antegrade Intramedullary Nailing of the Humeral Diaphysis. Orthopedics, 16, 1219-1223.

[8] Henley, M.B., Chapman, J.R. and Claudi, B.F. (1992) Closed Retrograde Hackethal Nail Stabilization of Humeral Shaft Fractures. Journal of Orthopaedic Trauma, 6, 18-24.

[9] Rommens, P.M., Verbruggen, J. and Broos, P.L. (1995) Retrograde Locked Nailing of Humeral Shaft Fractures. A Review of 39 Patients. Journal of Bone \& Joint Surgery, British Volume, 77, 84-89.

[10] Blum, J., Rommens, P.M. and Janzing, H. (1997) The Unreamed Humeral Nail—A Biological Osteosynthesis of the Upper arm. Acta Chirurgica Belgica, 97, 184-189.

[11] Garnavos, C. (2005) Humeral Nails: When to Choose What and How to Use. Current Orthopaedics, 19, $294-304$. http://dx.doi.org/10.1016/j.cuor.2005.06.003

[12] Garnavos, C. (2011) Diaphyseal Humeral Fractures and Intramedullary Nailing: Can We Improve Outcomes? Indian Journal of Orthopaedics, 45, 208-215. http://dx.doi.org/10.4103/0019-5413.67117

[13] Garnavos, C. (2001) Intramedullary Nailing for Humeral Shaft Fractures: The Misunderstood Poor Relative. Current Orthopaedics, 15, 68-75. http://dx.doi.org/10.1054/cuor.2001.0166

[14] Gierer, P., Scholz, M., Beck, M., Schaser, K.D., Vollmar, B., Mittlmeier, T. and Gradl, G. (2009) Microcirculatory Sequelae of the Rotator Cuff after Antegrade Nailing in Proximal Humerus Fracture. Archives of Orthopaedic and Trauma Surgery, 130, 687-691. http://dx.doi.org/10.1007/s00402-009-0956-z

[15] Euler, S.A., Hengg, C., Kolp, D., Wambacher, M. and Kralinger, F. (2014) Lack of Fifth Anchoring Point and Violation of the Insertion of the Rotator Cuff during Antegrade Humeralnailing: Pitfalls in Straight Antegrade Humeral Nailing. Bone \& Joint Journal, 96, 249-253. http://dx.doi.org/10.1302/0301-620X.96B2.31293

[16] Foster, R.J., Dixon Jr., G.L., Bach, A.W., et al. (1985) Internal Fixation of Fractures and Non-Unions of the Humeral Shaft. Indications and Results in a Multi-Center Study. Journal of Bone \& Joint Surgery, American Volume, 67, 857864.

[17] Brumback, R., Bose, M.J. and Poka, A. (1986) Intramedullary Stabilization of Humeral Shaft Fractures in Patients with Multiple Trauma. Journal of Bone \& Joint Surgery, American Volume, 68A, 960.

[18] Farragos, A.F., Schemitsch, E.H. and McKee, M.D. (1999) Complications of Intramedullary Nailing for Fractures of the Humeral Shaft: A Review. Journal of Orthopaedic Trauma, 13, 235-326. http://dx.doi.org/10.1097/00005131-199905000-00006

[19] Dimakopoulos, P., Papadopoulos, A.X., Papas, M. and Panagopoulos, A. (2005) Lambiris E Modified Extra RotatorCuff Entry Point in Antegrade Humeral Nailing. Archives of Orthopaedic and Trauma Surgery, 125, 27-32. http://dx.doi.org/10.1007/s00402-004-0757-3 